\title{
Non-Archimedean and random HUR-approximation of a Cauchy-Jensen additive mapping
}

\author{
Choonkil Park', Hassan Azadi Kenary² ${ }^{2^{*}}$ and Najmeh Sahami
}

\author{
*Correspondence: azadi@yu.ac.ir \\ 2Department of Mathematics, Beyza \\ Branch, Islamic Azad University, \\ Beyza, Iran \\ Full list of author information is \\ available at the end of the article
}

\begin{abstract}
In this paper, using the fixed point and direct methods, we prove the Hyers-Ulam-Rassias approximation (briefly, HUR-approximation) of a Cauchy-Jensen additive (briefly, CJA) functional equation in various normed spaces.

MSC: Primary 39B52; 39B82; 47H10; 46S10

Keywords: HUR-approximation; non-Archimedean normed space; random normed spaces; direct method; fixed point method
\end{abstract}

\section{Introduction}

A classical question in the theory of functional equations is the following: When is it true that a function which approximately satisfies a functional equation must be close to an exact solution of the equation? If the problem accepts a solution, we say that the equation is stable. The first stability problem concerning group homomorphisms was raised by Ulam [1] in 1940. In 1941, Hyers [2] gave a positive answer to the above question for additive groups under the assumption that the groups are Banach spaces. Aoki [3] proved a generalization of Hyers' theorem for additive mappings and Rassias [4] proved a generalization of Hyers' theorem for linear mappings.

Theorem 1.1 (ThM Rassias) Let $f: E \rightarrow E^{\prime}$ be a mapping from a normed vector space $E$ into a Banach space $E^{\prime}$ subject to the inequality $\|f(x+y)-f(x)-f(y)\| \leq \varepsilon\left(\|x\|^{p}+\|y\|^{p}\right)$, for all $x, y \in E$, where $\varepsilon$ and $p$ are constants with $\varepsilon>0$ and $0 \leq p<1$. Then the limit $L(x)=$ $\lim _{n \rightarrow \infty} \frac{f\left(2^{n} x\right)}{2^{n}}$ exists, for all $x \in E$, and $L: E \rightarrow E^{\prime}$ is the unique additive mapping which satisfies

$$
\|f(x)-L(x)\| \leq \frac{2 \varepsilon}{2-2^{p}}\|x\|^{p}
$$

for all $x \in E$. Also, iffor each $x \in E$ the function $f(t x)$ is continuous in $t \in \mathbb{R}$, then $L$ is linear.

This new concept is known as a the Hyers-Ulam stability or the Hyers-Ulam-Rassias stability of functional equations. Furthermore, in 1994, a generalization of Rassias' theorem was obtained by Gávruta [5] by replacing the bound $\varepsilon\left(\|x\|^{p}+\|y\|^{p}\right)$ by a general control function $\varphi(x, y)$.

\section{Springer}

O2014 Park et al.; licensee Springer. This is an Open Access article distributed under the terms of the Creative Commons Attribution License (http://creativecommons.org/licenses/by/2.0), which permits unrestricted use, distribution, and reproduction in any medium, provided the original work is properly cited. 
In 1983, a generalized Hyers-Ulam stability problem for the quadratic functional equation was proved by Skof [6] for mappings $f: X \rightarrow Y$, where $X$ is a normed space and $Y$ is a Banach space. In 1984, Cholewa [7] noticed that the theorem of Skof is still true if the relevant domain $X$ is replaced by an Abelian group and, in 2002, Czerwik [8] proved the generalized Hyers-Ulam stability of the quadratic functional equation. The readers are referred to [9-29] and references therein for detailed information on stability of functional equations.

In 1897, Hensel [30] has introduced a normed space which does not have the Archimedean property. It turned out that non-Archimedean spaces have many nice applications (see [31-35]).

Definition 1.1 By a non-Archimedean field we mean a field $\mathbb{K}$ equipped with a function (valuation) $|\cdot|: \mathbb{K} \rightarrow[0, \infty)$ such that, for all $r, s \in \mathbb{K}$, the following conditions hold: (a) $|r|=$ 0 if and only if $r=0$; (b) $|r s|=|r||s|$; (c) $|r+s| \leq \max \{|r|,|s|\}$.

Clearly, by (b), $|1|=|-1|=1$ and so, by induction, it follows from (c) that $|n| \leq 1$, for all $n \geq 1$.

Definition 1.2 Let $X$ be a vector space over a scalar field $\mathbb{K}$ with a non-Archimedean non-trivial valuation $|\cdot|$.

(1) A function $\|\cdot\|: X \rightarrow \mathbb{R}$ is a non-Archimedean norm (valuation) if it satisfies the following conditions: (a) $\|x\|=0$ if and only if $x=0$, for all $x \in X$; (b) $\|r x\|=|r|\|x\|$, for all $r \in \mathbb{K}$ and $x \in X$; (c) the strong triangle inequality (ultra-metric) holds, that is, $\|x+y\| \leq \max \{\|x\|,\|y\|\}$, for all $x, y \in X$.

(2) The space $(X,\|\cdot\|)$ is called a non-Archimedean normed space (briefly, NAN-space).

Note that $\left\|x_{n}-x_{m}\right\| \leq \max \left\{\left\|x_{j+1}-x_{j}\right\|: m \leq j \leq n-1\right\}$, for all $m, n \in \mathbb{N}$ with $n>m$.

Definition 1.3 Let $(X,\|\cdot\|)$ be a non-Archimedean normed space.

(a) A sequence $\left\{x_{n}\right\}$ is a Cauchy sequence in $X$ if $\left\{x_{n+1}-x_{n}\right\}$ converges to zero in $X$.

(b) The non-Archimedean normed space $(X,\|\cdot\|)$ is said to be complete if every Cauchy sequence in $X$ is convergent.

The most important examples of non-Archimedean spaces are $p$-adic numbers. A key property of $p$-adic numbers is that they do not satisfy the Archimedean axiom: for all $x, y>0$, there exists a positive integer $n$ such that $x<n y$.

Example 1.1 Fix a prime number $p$. For any nonzero rational number $x$, there exists a unique positive integer $n_{x}$ such that $x=\frac{a}{b} p^{n_{x}}$, where $a$ and $b$ are positive integers not divisible by $p$. Then $|x|_{p}:=p^{-n_{x}}$ defines a non-Archimedean norm on $\mathbb{Q}$. The completion of $\mathbb{Q}$ with respect to the metric $d(x, y)=|x-y|_{p}$ is denoted by $\mathbb{Q}_{p}$, which is called the $p$-adic number field. In fact, $\mathbb{Q}_{p}$ is the set of all formal series $x=\sum_{k \geq n_{x}}^{\infty} a_{k} p^{k}$, where $\left|a_{k}\right| \leq p-1$. The addition and multiplication between any two elements of $\mathbb{Q}_{p}$ are defined naturally. The norm $\left|\sum_{k \geq n_{x}}^{\infty} a_{k} p^{k}\right|_{p}=p^{-n_{x}}$ is a non-Archimedean norm on $\mathbb{Q}_{p}$ and $\mathbb{Q}_{p}$ is a locally compact field.

In Section 3, we adopt the usual terminology, notions and conventions of the theory of random normed spaces as in [36]. Throughout this paper, let $\Delta^{+}$denote the set of all 
probability distribution functions $F: \mathbb{R} \cup[-\infty,+\infty] \rightarrow[0,1]$ such that $F$ is left-continuous and nondecreasing on $\mathbb{R}$ and $F(0)=0, F(+\infty)=1$. It is clear that the set $D^{+}=\left\{F \in \Delta^{+}\right.$: $\left.l^{-} F(-\infty)=1\right\}$, where $l^{-} f(x)=\lim _{t \rightarrow x^{-}} f(t)$, is a subset of $\Delta^{+}$. The set $\Delta^{+}$is partially ordered by the usual point-wise ordering of functions, that is, $F \leq G$ if and only if $F(t) \leq G(t)$, for all $t \in \mathbb{R}$. For any $a \geq 0$, the element $H_{a}(t)$ of $D^{+}$is defined by

$$
H_{a}(t)= \begin{cases}0, & \text { if } t \leq a \\ 1, & \text { if } t>a\end{cases}
$$

We can easily show that the maximal element in $\triangle^{+}$is the distribution function $H_{0}(t)$.

Definition 1.4 A function $T:[0,1]^{2} \rightarrow[0,1]$ is a continuous triangular norm (briefly, a $t$ norm) if $T$ satisfies the following conditions: (a) $T$ is commutative and associative; (b) $T$ is continuous; (c) $T(x, 1)=x$, for all $x \in[0,1]$; (d) $T(x, y) \leq T(z, w)$ whenever $x \leq z$ and $y \leq w$, for all $x, y, z, w \in[0,1]$.

Three typical examples of continuous $t$-norms are as follows: $T_{P}(x, y)=x y, T_{\max }(x, y)=$ $\max \{a+b-1,0\}, T_{M}(x, y)=\min (a, b)$. Recall that, if $T$ is a $t$-norm and $\left\{x_{n}\right\}$ is a sequence in $[0,1]$, then $T_{i=1}^{n} x_{i}$ is defined recursively by $T_{i=1}^{1} x_{1}=x_{1}$ and $T_{i=1}^{n} x_{i}=T\left(T_{i=1}^{n-1} x_{i}, x_{n}\right)$, for all $n \geq 2 . T_{i=n}^{\infty} x_{i}$ is defined by $T_{i=1}^{\infty} x_{n+i}$.

Definition 1.5 A random normed space (briefly, $R N$-space) is a triple $(X, \mu, T)$, where $X$ is a vector space, $T$ is a continuous $t$-norm and $\mu: X \rightarrow D^{+}$is a mapping such that the following conditions hold:

(a) $\mu_{x}(t)=H_{0}(t)$, for all $t>0$ if and only if $x=0$;

(b) $\mu_{\alpha x}(t)=\mu_{x}\left(\frac{t}{|\alpha|}\right)$, for all $\alpha \in \mathbb{R}$ with $\alpha \neq 0, x \in X$ and $t \geq 0$;

(c) $\mu_{x+y}(t+s) \geq T\left(\mu_{x}(t), \mu_{y}(s)\right)$, for all $x, y \in X$ and $t, s \geq 0$.

Every normed space $(X,\|\cdot\|)$ defines a random normed space $\left(X, \mu, T_{M}\right)$, where $\mu_{u}(t)=$ $\frac{t}{t+\|u\|}$, for all $t>0$ and $T_{M}$ is the minimum $t$-norm. This space $X$ is called the induced random normed space.

If the $t$-norm $T$ is such that $\sup _{0<a<1} T(a, a)=1$, then every $R N$-space $(X, \mu, T)$ is a metrizable linear topological space with the topology $\tau$ (called the $\mu$-topology or the $(\varepsilon, \delta)$ topology, where $\varepsilon>0$ and $\lambda \in(0,1))$ induced by the base $\{U(\varepsilon, \lambda)\}$ of neighborhoods of $\theta$, where

$$
U(\varepsilon, \lambda)=\left\{x \in X: \mu_{x}(\varepsilon)>1-\lambda\right\} .
$$

Definition 1.6 Let $(X, \mu, T)$ be an RN-space.

(a) A sequence $\left\{x_{n}\right\}$ in $X$ is said to be convergent to a point $x \in X$ (write $x_{n} \rightarrow x$ as $n \rightarrow \infty)$ if $\lim _{n \rightarrow \infty} \mu_{x_{n}-x}(t)=1$, for all $t>0$.

(b) A sequence $\left\{x_{n}\right\}$ in $X$ is called a Cauchy sequence in $X$ if $\lim _{n \rightarrow \infty} \mu_{x_{n}-x_{m}}(t)=1$, for all $t>0$.

(c) The $R N$-space $(X, \mu, T)$ is said to be complete if every Cauchy sequence in $X$ is convergent. 
Theorem 1.2 If $(X, \mu, T)$ is RN-space and $\left\{x_{n}\right\}$ is a sequence such that $x_{n} \rightarrow x$, then $\lim _{n \rightarrow \infty} \mu_{x_{n}}(t)=\mu_{x}(t)$.

Definition 1.7 Let $X$ be a set. A function $d: X \times X \rightarrow[0, \infty]$ is called a generalized metric on $X$ if $d$ satisfies the following conditions:

(a) $d(x, y)=0$ if and only if $x=y$, for all $x, y \in X$;

(b) $d(x, y)=d(y, x)$, for all $x, y \in X$;

(c) $d(x, z) \leq d(x, y)+d(y, z)$, for all $x, y, z \in X$.

Theorem $1.3([37,38])$ Let $(X, d)$ be a complete generalized metric space and $J: X \rightarrow X$ be a strictly contractive mapping with Lipschitz constant $L<1$. Then, for all $x \in X$, either $d\left(J^{n} x, J^{n+1} x\right)=\infty$, for all nonnegative integers $n$, or there exists a positive integer $n_{0}$ such that

(a) $d\left(J^{n} x, J^{n+1} x\right)<\infty$, for all $n_{0} \geq n_{0}$;

(b) the sequence $\left\{J^{n} x\right\}$ converges to a fixed point $y^{*}$ of $J$;

(c) $y^{*}$ is the unique fixed point of $J$ in the set $Y=\left\{y \in X: d\left(J^{n_{0}} x, y\right)<\infty\right\}$;

(d) $d\left(y, y^{*}\right) \leq \frac{d(y, J y)}{1-L}$, for all $y \in Y$.

In this paper, using the fixed point and direct methods, we prove the HUR-approximation of the following CJA functional equation:

$$
2 f\left(\frac{x+y+z}{2}\right)=f(x)+f(y)+f(z)
$$

in various normed spaces.

\section{NAN-stability}

In this section, we deal with the stability problem for the Cauchy-Jensen additive functional equation (1.1) in non-Archimedean normed spaces.

Theorem 2.1 Let $X$ be a non-Archimedean normed space and $Y$ is a complete nonArchimedean space. Let $\varphi: X^{3} \rightarrow[0, \infty)$ be a function such that there exists an $\alpha<1$ with

$$
\varphi\left(\frac{x}{2}, \frac{y}{2}, \frac{z}{2}\right) \leq \frac{\alpha \varphi(x, y, z)}{|2|}
$$

for all $x, y, z \in X$. Let $f: X \rightarrow Y$ be a mapping satisfying

$$
\left\|2 f\left(\frac{x+y+z}{2}\right)-f(x)-f(y)-f(z)\right\|_{Y} \leq \varphi(x, y, z)
$$

for all $x, y, z \in X$. Then there exists a unique additive mapping $\mathfrak{\Im}: X \rightarrow Y$ such that

$$
\|f(x)-\Im(x)\|_{Y} \leq \alpha \varphi(x, 2 x, x)(|2|-|2| \alpha)^{-1}
$$

for all $x \in X$. 
Proof Putting $y=2 x$ and $z=x$ in (2.1), we get $\|f(2 x)-2 f(x)\|_{Y} \leq \varphi(x, 2 x, x)$, for all $x \in X$. So

$$
\left\|f(x)-2 f\left(\frac{x}{2}\right)\right\|_{Y} \leq|2|^{-1} \alpha \varphi(x, 2 x, x)
$$

for all $x \in X$. Consider the set $S:=\{h: X \rightarrow Y\}$ and introduce the generalized metric on $S$ :

$$
d(g, h)=\inf _{\mu \in(0,+\infty)}\|g(x)-h(x)\|_{Y} \leq \mu \varphi(x, 2 x, x)
$$

for all $x \in X$, where, as usual, $\inf \phi=+\infty$. It is easy to show that $(S, d)$ is complete (see [39]). Now we consider the linear mapping $J: S \rightarrow S$ such that $g g(x):=2 g\left(\frac{x}{2}\right)$, for all $x \in X$. Let $g, h \in S$ be given such that $d(g, h)=\varepsilon$. Then $\|g(x)-h(x)\|_{Y} \leq \varepsilon \varphi(x, 2 x, x)$, for all $x \in X$. Hence

$$
\|J g(x)-J h(x)\|_{Y}=\left\|2 g\left(\frac{x}{2}\right)-2 h\left(\frac{x}{2}\right)\right\|_{Y} \leq \alpha \cdot \varepsilon \varphi(x, 2 x, x),
$$

for all $x \in X$. So $d(g, h)=\varepsilon$ implies that $d(J g, J h) \leq \alpha \varepsilon$. This means that $d(J g, J h) \leq \alpha d(g, h)$, for all $g, h \in S$. It follows from (2.4) that $d(f, J f) \leq|2|^{-1} \alpha$. By Theorem 1.3, there exists a mapping $\mathfrak{s}: X \rightarrow Y$ satisfying the following:

(1) $\Im$ is a fixed point of $J$, i.e.,

$$
\Im(x)=2 \Im\left(\frac{x}{2}\right),
$$

for all $x \in X$. The mapping $\Im$ is a unique fixed point of $J$ in the set $M=\{g \in S: d(h, g)<\infty\}$. This implies that $\mathfrak{I}$ is a unique mapping satisfying (2.5) such that there exists a $\mu \in(0, \infty)$ satisfying $\|f(x)-\mathfrak{\Im}(x)\|_{Y} \leq \mu \varphi(x, 2 x, x)$, for all $x \in X$;

(2) $d\left(J^{n} f, \Im\right) \rightarrow 0$ as $n \rightarrow \infty$. This implies the equality

$$
\lim _{n \rightarrow \infty} 2^{n} f\left(\frac{x}{2^{n}}\right)=\Im(x),
$$

for all $x \in X$;

(3) $d(f, \Im) \leq \frac{d(f, f f)}{1-\alpha}$, which implies the inequality $d(f, \Im) \leq \alpha(|2|-|2| \alpha)^{-1}$. This implies that the inequalities (2.3) holds. It follows from (2.1) and (2.2) that

$$
\begin{aligned}
& \left\|2 \Im\left(\frac{x+y+z}{2}\right)-\Im(x)-\Im(y)-\Im(z)\right\|_{Y} \\
& \quad=\lim _{n \rightarrow \infty} \frac{\left\|2 f\left(\frac{x+y+z}{2^{n+1}}\right)-f\left(\frac{x}{2^{n}}\right)-f\left(\frac{y}{2^{n}}\right)-f\left(\frac{z}{2^{n}}\right)\right\|_{Y}}{|2|^{-n}} \\
& \quad \leq \lim _{n \rightarrow \infty} \alpha^{n} \varphi(x, y, z)=0,
\end{aligned}
$$

for all $x, y, z \in X$. So $2 \Im\left(\frac{x+y+z}{2}\right)=\Im(x)+\Im(y)+\Im(z)$, for all $x, y, z \in X$. Hence $\Im: X \rightarrow Y$ is an CJA mapping and we get the desired results. 
Corollary 2.1 Let $\theta$ be a positive real number and $r$ is a real number with $0<r<1$. Let $f: X \rightarrow Y$ be a mapping satisfying

$$
\left\|2 f\left(\frac{x+y+z}{2}\right)-f(x)-f(y)-f(z)\right\|_{Y} \leq \theta\left(\|x\|^{r}+\|y\|^{r}+\|z\|^{r}\right)
$$

for all $x, y, z \in X$. Then there exists a unique CJA mapping $\Im: X \rightarrow Y$ such that

$$
\|f(x)-\Im(x)\|_{Y} \leq|2| \theta\left(2+|2|^{r}\right)\left(|2|^{r+1}-|2|^{2}\right)^{-1}\|x\|^{r},
$$

for all $x \in X$.

Proof The proof follows from Theorem 2.1 by taking $\varphi(x, y, z)=\left(\|x\|^{r}+\|y\|^{r}+\|z\|^{r}\right)$, for all $x, y, z \in X$. Then we can choose $\alpha=|2|^{1-r}$ and we get the desired result.

Theorem 2.2 Let $X$ be a non-Archimedean normed space and $Y$ is a complete nonArchimedean space. Let $\varphi: X^{3} \rightarrow[0, \infty)$ be a function such that there exists an $\alpha<1$ with $\varphi(x, y, z) \leq|2| \alpha \varphi\left(\frac{x}{2}, \frac{y}{2}, \frac{z}{2}\right)$, for all $x, y, z \in X$. Let $f: X \rightarrow Y$ be a mapping satisfying (2.2). Then there exists a unique CJA mapping $\Im: X \rightarrow Y$ such that

$$
\|f(x)-\Im(x)\|_{Y} \leq \varphi(x, 2 x, x)(|2|-|2| \alpha)^{-1}
$$

for all $x \in X$.

Proof Let $(S, d)$ be the generalized metric space defined in the proof of Theorem 2.1. Now we consider the linear mapping $J: S \rightarrow S$ such that $g(x):=\frac{g(2 x)}{2}$, for all $x \in X$. Let $g, h \in S$ be given such that $d(g, h)=\varepsilon$. Then $\|g(x)-h(x)\|_{Y} \leq \varepsilon \varphi(x, 2 x, x)$, for all $x \in X$. Hence

$$
\|J g(x)-J h(x)\|_{Y}=\left\|\frac{g(2 x)}{2}-\frac{h(2 x)}{2}\right\|_{Y} \leq \frac{|2| \alpha \cdot \varepsilon \varphi(x, 2 x, x)}{|2|},
$$

for all $x \in X$. So $d(g, h)=\varepsilon$ implies that $d(J g, J h) \leq \alpha \varepsilon$. This means that $d(J g, J h) \leq \alpha d(g, h)$, for all $g, h \in S$. It follows from (2.4) that $d(f, J f) \leq|2|^{-1}$. By Theorem 1.3, there exists a mapping $\mathfrak{s}: X \rightarrow Y$ satisfying the following:

(1) $\Im$ is a fixed point of $J$, i.e.,

$$
\frac{\Im(2 x)}{2}=\Im(x),
$$

for all $x \in X$. The mapping $\Im$ is a unique fixed point of $J$ in the set $M=\{g \in S: d(h, g)<\infty\}$. This implies that $\Im$ is a unique mapping satisfying (2.9) such that there exists a $\mu \in(0, \infty)$ satisfying $\|f(x)-\Im(x)\|_{Y} \leq \mu \varphi(x, 2 x, x)$, for all $x \in X$;

(2) $d\left(J^{n} f, \Im\right) \rightarrow 0$ as $n \rightarrow \infty$. This implies the equality $\lim _{n \rightarrow \infty} \frac{f\left(2^{n} x\right)}{2^{n}}=\Im(x)$, for all $x \in X$;

(3) $d(f, \Im) \leq \frac{d(f, J f)}{1-\alpha}$, which implies the inequality $d(f, \Im) \leq(|2|-|2| \alpha)^{-1}$. This implies that the inequalities (2.8) holds. The rest of the proof is similar to the proof of Theorem 2.1. 
Corollary 2.2 Let $\theta$ be a positive real number and $r$ is a real number with $r>1$. Let $f$ : $X \rightarrow Y$ be a mapping satisfying (2.7). Then there exists a unique CJA mapping $\Im: X \rightarrow Y$ such that

$$
\|f(x)-\Im(x)\|_{Y} \leq \theta\left(2+|2|^{r}\right)\left(|2|-|2|^{r}\right)^{-1}\|x\|^{r},
$$

for all $x \in X$.

Proof The proof follows from Theorem 2.2 by taking $\varphi(x, y, z)=\left(\|x\|^{r}+\|y\|^{r}+\|z\|^{r}\right)$, for all $x, y, z \in X$. Then we can choose $\alpha=|2|^{r-1}$ and we get the desired result.

Theorem 2.3 Let $G$ be an additive semigroup and $X$ is a non-Archimedean Banach space. Assume that $\lambda: G^{3} \rightarrow[0,+\infty)$ be a function such that

$$
\lim _{n \rightarrow \infty}|2|^{n} \lambda\left(\frac{x}{2^{n}}, \frac{y}{2^{n}}, \frac{z}{2^{n}}\right)=0,
$$

for all $x, y, z \in G$. Suppose that, for any $x \in G$, the limit

$$
£(x)=\lim _{n \rightarrow \infty} \max _{0 \leq k<n}|2|^{k} \lambda\left(\frac{x}{2^{k+1}}, \frac{x}{2^{k}}, \frac{x}{2^{k+1}}\right)
$$

exists and $f: G \rightarrow X$ be a mapping satisfying

$$
\left\|2 f\left(\frac{x+y+z}{2}\right)-f(x)-f(y)-f(z)\right\|_{X} \leq \lambda(x, y, z)
$$

Then the limit $\Im(x):=\lim _{n \rightarrow \infty} 2^{n} f\left(\frac{x}{2^{n}}\right)$ exists, for all $x \in G$, and defines an CJA mapping $\Im: G \rightarrow X$ such that

$$
\|f(x)-\Im(x)\| \leq \mathfrak{f}(x)
$$

Moreover, if $\lim _{j \rightarrow \infty} \lim _{n \rightarrow \infty} \max _{j \leq k<n+j}|2|^{k} \lambda\left(\frac{x}{2^{k+1}}, \frac{x}{2^{k}}, \frac{x}{2^{k+1}}\right)=0$ then $\Im$ is the unique CJA mapping satisfying (2.13).

Proof Putting $y=2 x$ and $z=x$ in (2.12), we get

$$
\|f(2 x)-2 f(x)\|_{Y} \leq \lambda(x, 2 x, x)
$$

for all $x \in G$. Replacing $x$ by $\frac{x}{2^{n+1}}$ in (2.14), we obtain

$$
\left\|2^{n+1} f\left(\frac{x}{2^{n+1}}\right)-2^{n} f\left(\frac{x}{2^{n}}\right)\right\| \leq|2|^{n} \lambda\left(\frac{x}{2^{n+1}}, \frac{x}{2^{n}}, \frac{x}{2^{n+1}}\right) .
$$

Thus, it follows from (2.10) and (2.15) that the sequence $\left\{2^{n} f\left(\frac{x}{2^{n}}\right)\right\}_{n \geq 1}$ is a Cauchy sequence. Since $X$ is complete, it follows that $\left\{2^{n} f\left(\frac{x}{2^{n}}\right)\right\}_{n \geq 1}$ is convergent. Set $\Im(x):=\lim _{n \rightarrow \infty} 2^{n} f\left(\frac{x}{2^{n}}\right)$. By induction on $n$, one can show that

$$
\left\|2^{n} f\left(\frac{x}{2^{n}}\right)-f(x)\right\| \leq \max _{0 \leq k<n}|2|^{k} \lambda\left(\frac{x}{2^{k+1}}, \frac{x}{2^{k}}, \frac{x}{2^{k+1}}\right),
$$


for all $n \geq 1$ and $x \in G$. By taking $n \rightarrow \infty$ in (2.16) and using (2.11), one obtains (2.13). By (2.10) and (2.12), we get

$$
\begin{aligned}
& \| 2 \Im\left(\frac{x+y+z}{2}\right)-\Im(x)-\Im(y)-\Im(z) \| \\
& \quad \lim _{n \rightarrow \infty}|2|^{n}\left\|2 f\left(\frac{x+y+z}{2^{n+1}}\right)-f\left(\frac{x}{2^{n}}\right)-f\left(\frac{y}{2^{n}}\right)-f\left(\frac{z}{2^{n}}\right)\right\| \\
& \quad \leq \lim _{n \rightarrow \infty}|2|^{n} \lambda\left(\frac{x}{2^{n}}, \frac{y}{2^{n}}, \frac{z}{2^{n}}\right)=0,
\end{aligned}
$$

for all $x, y, z \in X$. So

$$
\Im\left(\frac{x+y+z}{2}\right)=\Im(x)+\Im(y)+\Im(z) .
$$

Letting $x=y=z=0$ in (2.17), we get $\Im(0)=0$. Letting $z=x+y$ in (2.17), we get $\Im(x+y)=$ $\mathfrak{I}(x)+\mathfrak{I}(y)$, for all $x, y \in X$. Hence the mapping $\mathfrak{\Im}: X \rightarrow Y$ is Cauchy additive.

To prove the uniqueness property of $\Im$, let $\Re$ be another mapping satisfying (2.13). Then we have

$$
\begin{aligned}
\|\Im(x)-\Re(x)\|_{X} & =\lim _{n \rightarrow \infty}|2|^{n}\left\|\Im\left(\frac{x}{2^{n}}\right)-\mathfrak{\Re}\left(\frac{x}{2^{n}}\right)\right\|_{X} \\
& \leq \lim _{k \rightarrow \infty}|2|^{n} \max \left\{\left\|\Im\left(\frac{x}{2^{n}}\right)-f\left(\frac{x}{2^{n}}\right)\right\|_{X},\left\|f\left(\frac{x}{2^{n}}\right)-\Re\left(\frac{x}{2^{n}}\right)\right\|_{X}\right\} \\
& \leq \lim _{j \rightarrow \infty} \lim _{n \rightarrow \infty} \max _{j \leq k<n+j}|2|^{k} \lambda\left(\frac{x}{2^{k+1}}, \frac{x}{2^{k}}, \frac{x}{2^{k+1}}\right)=0,
\end{aligned}
$$

for all $x \in G$. Therefore, $\Im=\Re$. This completes the proof.

Corollary 2.3 Let $\xi:[0, \infty) \rightarrow[0, \infty)$ be a function satisfying $\xi\left(\frac{t}{|2|}\right) \leq \xi\left(\frac{1}{|2|}\right) \xi(t), \xi\left(\frac{1}{|2|}\right)<$ $\frac{1}{|2|}$, for all $t \geq 0$. Assume that $\kappa>0$ and $f: G \rightarrow X$ be a mapping such that

$$
\left\|2 f\left(\frac{x+y+z}{2}\right)-f(x)-f(y)-f(z)\right\|_{Y} \leq \kappa(\xi(|x|)+\xi(|y|)+\xi(|z|))
$$

for all $x, y, z \in G$. Then there exists a unique CJA mapping $\Im: G \rightarrow X$ such that

$$
\|f(x)-\Im(x)\| \leq|2|^{-1}(2+|2|) \xi(|x|) .
$$

Proof If we define $\lambda: G^{3} \rightarrow[0, \infty)$ by $\lambda(x, y, z):=\kappa(\xi(|x|)+\xi(|y|)+\xi(|z|))$, then we have $\lim _{n \rightarrow \infty}|2|^{n} \lambda\left(\frac{x}{2^{n}}, \frac{y}{2^{n}}, \frac{z}{2^{n}}\right)=0$, for all $x, y, z \in G$. On the other hand, it follows that $£(x)=$ $|2|^{-1}(2+|2|) \xi(|x|)$ exists, for all $x \in G$. Also, we have

$$
\lim _{j \rightarrow \infty} \lim _{n \rightarrow \infty} \max _{j \leq k<n+j}|2|^{k} \lambda\left(\frac{x}{2^{k+1}}, \frac{x}{2^{k}}, \frac{x}{2^{k+1}}\right)=\lim _{j \rightarrow \infty}|2|^{j} \lambda\left(\frac{x}{2^{j+1}}, \frac{x}{2^{j}}, \frac{x}{2^{j+1}}\right)=0 .
$$

Thus, applying Theorem 2.3, we have the conclusion. This completes the proof. 
Theorem 2.4 Let $G$ be an additive semigroup and $X$ is a non-Archimedean Banach space. Assume that $\lambda: G^{3} \rightarrow[0,+\infty)$ be a function such that $\lim _{n \rightarrow \infty} \frac{\lambda\left(2^{n} x, 2^{n} y, 2^{n} z\right)}{2^{n}}=0$, for all $x, y, z \in G$. Suppose that, for any $x \in G$, the limit

$$
\mathfrak{f}(x)=\lim _{n \rightarrow \infty} \max _{0 \leq k<n} \frac{\lambda\left(2^{k} x, 2^{k+1} x, 2^{k} x\right)}{|2|^{k}}
$$

exists and $f: G \rightarrow X$ be a mapping satisfying (2.12). Then the limit $\Im(x):=\lim _{n \rightarrow \infty} \frac{f\left(2^{n} x\right)}{2^{n}}$ exists, for all $x \in G$, and

$$
\|f(x)-\Im(x)\| \leq \frac{\mathfrak{f}(x)}{|2|},
$$

for all $x \in$ G. Moreover, if $\lim _{j \rightarrow \infty} \lim _{n \rightarrow \infty} \max _{j \leq k<n+j} \frac{\lambda\left(2^{k} x, 2^{k+1} x, 2^{k} x\right)}{|2|^{k}}=0$, then $\Im$ is the unique CJA mapping satisfying (2.19).

Proof It follows from (2.14) that

$$
\left\|f(x)-\frac{f(2 x)}{2}\right\|_{X} \leq \frac{\lambda(x, 2 x, x)}{|2|},
$$

for all $x \in G$. Replacing $x$ by $2^{n} x$ in (2.20), we obtain

$$
\left\|\frac{f\left(2^{n} x\right)}{2^{n}}-\frac{f\left(2^{n+1} x\right)}{2^{n+1}}\right\|_{X} \leq \frac{\lambda\left(2^{n} x, 2^{n+1} x, 2^{n} x\right)}{|2|^{n+1}} .
$$

Thus it follows from (2.21) that the sequence $\left\{\frac{f\left(2^{n} x\right)}{2^{n}}\right\}_{n \geq 1}$ is convergent. Set $\Im(x):=$ $\lim _{n \rightarrow \infty} \frac{f\left(2^{n} x\right)}{2^{n}}$. On the other hand, it follows from $(2.21)$ that

$$
\begin{aligned}
\left\|\frac{f\left(2^{p} x\right)}{2^{p}}-\frac{f\left(2^{q} x\right)}{2^{q}}\right\| & =\left\|\sum_{k=p}^{q-1} \frac{f\left(2^{k+1} x\right)}{2^{k+1}}-\frac{f\left(2^{k} x\right)}{2^{k}}\right\| \leq \max _{p \leq k<q}\left\{\left\|\frac{f\left(2^{k+1} x\right)}{2^{k+1}}-\frac{f\left(2^{k} x\right)}{2^{k}}\right\|\right\} \\
& \leq \frac{1}{|2|} \max _{p \leq k<q} \frac{\lambda\left(2^{k} x, 2^{k+1} x, 2^{k} x\right)}{|2|^{k}},
\end{aligned}
$$

for all $x \in G$ and $p, q \geq 0$ with $q>p \geq 0$. Letting $p=0$, taking $q \rightarrow \infty$ in the last inequality and using (2.18), we obtain (2.19).

The rest of the proof is similar to the proof of Theorem 2.3. This completes the proof.

Corollary 2.4 Let $\xi:[0, \infty) \rightarrow[0, \infty)$ be a function satisfying $\xi(|2| t) \leq \xi(|2|) \xi(t), \xi(|2|)<$ $|2|$, for all $t \geq 0$. Let $\kappa>0$ and $f: G \rightarrow X$ be a mapping satisfying

$$
\left\|2 f\left(\frac{x+y+z}{2}\right)-f(x)-f(y)-f(z)\right\| \leq \kappa(\xi(|x|) \cdot \xi(|y|) \cdot \xi(|z|)),
$$

for all $x, y, z \in G$. Then there exists a unique CJA mapping $\Im: G \rightarrow X$ such that

$$
\|f(x)-\Im(x)\| \leq \kappa \xi(|x|)^{3} .
$$


Proof If we define $\lambda: G^{3} \rightarrow[0, \infty)$ by $\lambda(x, y, z):=\kappa(\xi(|x|) \cdot \xi(|y|) \cdot \xi(|z|))$ and apply Theorem 2.4, then we get the conclusion.

\section{RNS-stability}

In this section, using the fixed point and direct methods, we prove the HUR-approximation of the functional equation (1.1) in random normed spaces.

Theorem 3.1 Let $X$ be a real linear space, $\left(Z, \mu^{\prime}\right.$, min) be an $R N$-space and $\varphi: X^{3} \rightarrow Z$ be a function such that there exists $0<\alpha<\frac{1}{2}$ such that

$$
\mu_{\varphi\left(\frac{x}{2}, \frac{y}{2}, \frac{z}{2}\right)}^{\prime}(t) \geq \mu_{\varphi(x, y, z)}^{\prime}\left(\frac{t}{\alpha}\right)
$$

for all $x, y, z \in X$ and $t>0$ and $\lim _{n \rightarrow \infty} \mu_{\varphi\left(\frac{x}{2^{n}}, \frac{y}{2^{n}}, \frac{z}{2^{n}}\right)}^{\prime}\left(\frac{t}{2^{n}}\right)=1$, for all $x, y, z \in X$ and $t>0$. Let $(Y, \mu, \min )$ be a complete $R N$-space. If $: X \rightarrow Y$ be a mapping such that

$$
\mu_{2 f\left(\frac{x+y+z}{2}\right)-f(x)-f(y)-f(z)}(t) \geq \mu_{\varphi(x, y, z)}^{\prime}(t)
$$

for all $x, y, z \in X$ and $t>0$. Then the limit $\Im(x)=\lim _{n \rightarrow \infty} 2^{n} f\left(\frac{x}{2^{n}}\right)$ exists, for all $x \in X$, and defines a unique CJA mapping $\mathfrak{s}: X \rightarrow Y$ such that

$$
\mu_{f(x)-A(x)}(t) \geq \mu_{\varphi(x, 2 x, x)}^{\prime}\left(\frac{(1-2 \alpha) t}{\alpha}\right)
$$

for all $x \in X$ and $t>0$.

Proof Putting $y=2 x$ and $z=x$ in (3.2), we see that

$$
\mu_{f(2 x)-2 f(x)}(t) \geq \mu_{\varphi(x, 2 x, x)}^{\prime}(t) .
$$

Replacing $x$ by $\frac{x}{2}$ in (3.4), we obtain

$$
\mu_{2 f\left(\frac{x}{2}\right)-f(x)}(t) \geq \mu_{\varphi\left(\frac{x}{2}, x, \frac{x}{2}\right)}^{\prime}(t) \geq \mu_{\varphi(x, 2 x, x)}^{\prime}\left(\frac{t}{\alpha}\right)
$$

for all $x \in X$. Replacing $x$ by $\frac{x}{2^{n}}$ in (3.5) and using (3.1), we obtain

$$
\mu_{2^{n+1} f\left(\frac{x}{2^{n+1}}\right)-2^{n} f\left(\frac{x}{2^{n}}\right)}(t) \geq \mu_{\varphi\left(\frac{x}{2^{n+1}}, \frac{x}{2^{n}}, \frac{x}{2^{n+1}}\right)}^{\prime}\left(\frac{t}{2^{n}}\right) \geq \mu_{\varphi(x, 2 x, x)}^{\prime}\left(\frac{t}{2^{n} \alpha^{n+1}}\right)
$$

and so

$$
\begin{aligned}
\mu_{2^{n} f\left(\frac{x}{2^{n}}\right)-f(x)}\left(\sum_{k=0}^{n-1} 2^{k} \alpha^{k+1} t\right) & =\mu_{\sum_{k=0}^{n-1} 2^{k+1} f\left(\frac{x}{2^{k+1}}\right)-2^{k} f\left(\frac{x}{2^{k}}\right)}\left(\sum_{k=0}^{n-1} 2^{k} \alpha^{k+1} t\right) \\
& \geq T_{k=0}^{n-1}\left(\mu_{2^{k+1} f\left(\frac{x}{2^{k+1}}\right)-2^{k} f\left(\frac{x}{2^{k}}\right)}\left(2^{k} \alpha^{k+1} t\right)\right) \\
& \geq T_{k=0}^{n-1}\left(\mu_{\varphi(x, 2 x, x)}^{\prime}(t)\right)=\mu_{\varphi(x, 2 x, x)}^{\prime}(t) .
\end{aligned}
$$


This implies that

$$
\mu_{2^{n} f\left(\frac{x}{2^{n}}\right)-f(x)}(t) \geq \mu_{\varphi(x, 2 x, x)}^{\prime}\left(\frac{t}{\sum_{k=0}^{n-1} 2^{k} \alpha^{k+1}}\right) .
$$

Replacing $x$ by $\frac{x}{2^{p}}$ in (3.6), we obtain

$$
\mu_{2^{n+p} f\left(\frac{x}{\left.2^{n+p}\right)-2^{p}} f\left(\frac{x}{2^{p}}\right)\right.}(t) \geq \mu_{\varphi(x, 2 x, x)}^{\prime}\left(\frac{t}{\sum_{k=p}^{n+p-1} 2^{k} \alpha^{k+1}}\right) .
$$

Since $\lim _{p, n \rightarrow \infty} \mu_{\varphi(x, 2 x, x)}^{\prime}\left(\frac{t}{\sum_{k=p}^{n+p-1} 2^{k} \alpha^{k+1}}\right)=1$, it follows that $\left\{2^{n} f\left(\frac{x}{2^{n}}\right)\right\}_{n=1}^{\infty}$ is a Cauchy sequence in a complete $\mathrm{RN}$-space $(Y, \mu, \min )$ and so there exists a point $\Im(x) \in Y$ such that $\lim _{n \rightarrow \infty} 2^{n} f\left(\frac{x}{2^{n}}\right)=\Im(x)$. Fix $x \in X$ and put $p=0$ in (3.7) and so, for any $\varepsilon>0$,

$$
\mu_{\Im(x)-f(x)}(t+\varepsilon) \geq T\left(\mu_{\Im(x)-2^{n} f\left(\frac{x}{2^{n}}\right)}(\varepsilon), \mu_{\varphi(x, 2 x, x)}^{\prime}\left(\frac{t}{\sum_{k=0}^{n-1} 2^{k} \alpha^{k+1}}\right)\right) .
$$

Taking $n \rightarrow \infty$ in (3.8), we get $\mu_{\Im(x)-f(x)}(t+\varepsilon) \geq \mu_{\varphi(x, 2 x, x)}^{\prime}\left(\frac{(1-2 \alpha) t}{\alpha}\right)$. Since $\varepsilon$ is arbitrary, by taking $\varepsilon \rightarrow 0$ in the previous inequality, we get

$$
\mu_{\Im(x)-f(x)}(t) \geq \mu_{\varphi(x, 2 x, x)}^{\prime}\left(\frac{(1-2 \alpha) t}{\alpha}\right) .
$$

Replacing $x, y$ and $z$ by $\frac{x}{2^{n}}, \frac{y}{2^{n}}$ and $\frac{z}{2^{n}}$ in (3.2), respectively, we get

$$
\mu_{2^{n+1} f\left(\frac{x+y+z}{2^{n+1}}\right)-2^{n} f\left(\frac{x}{2^{n}}\right)-2^{n} f\left(\frac{y}{2^{n}}\right)-2^{n} f\left(\frac{z}{2^{n}}\right)}(t) \geq \mu_{\varphi\left(\frac{x}{2^{n}}, \frac{y}{2^{n}}, \frac{z}{2^{n}}\right)}^{\prime}\left(\frac{t}{2^{n}}\right),
$$

for all $x, y, z \in X$ and $t>0$. Since $\lim _{n \rightarrow \infty} \mu_{\varphi\left(\frac{x}{2^{n}}, \frac{y}{2^{n}}, \frac{z}{2^{n}}\right)}^{\prime}\left(\frac{t}{2^{n}}\right)=1$, we conclude that $\Im$ satisfies (1.1). On the other hand

$$
2 \Im\left(\frac{x}{2}\right)-\Im(x)=\lim _{n \rightarrow \infty} 2^{n+1} f\left(\frac{x}{2^{n+1}}\right)-\lim _{n \rightarrow \infty} 2^{n} f\left(\frac{x}{2^{n}}\right)=0 .
$$

This implies that $\mathfrak{s}: X \rightarrow Y$ is an CJA mapping. To prove the uniqueness of the CJA mapping $\mathfrak{\Im}$, assume that there exists another CJA mapping $\Re: X \rightarrow Y$ which satisfies (3.3). Then we have

$$
\begin{aligned}
\mu_{\Im(x)-\Re(x)}(t) & =\lim _{n \rightarrow \infty} \mu_{2^{n}\left(\frac{x}{2^{n}}\right)-2^{n} \Re\left(\frac{x}{2^{n}}\right)}(t) \\
& \geq \lim _{n \rightarrow \infty} \min \left\{\mu_{2^{n} \Im\left(\frac{x}{2^{n}}\right)-2^{n} f\left(\frac{x}{2^{n}}\right)}\left(\frac{t}{2}\right), \mu_{2^{n} f\left(\frac{x}{2^{n}}\right)-2^{n} \Re\left(\frac{x}{2^{n}}\right)}\left(\frac{t}{2}\right)\right\} \\
& \geq \lim _{n \rightarrow \infty} \mu_{\varphi\left(\frac{x}{2^{n}}, \frac{2 x}{2^{n}}, \frac{x}{2^{n}}\right)}^{\prime}\left(\frac{(1-2 \alpha) t}{2^{n}}\right) \geq \lim _{n \rightarrow \infty} \mu_{\varphi(x, 2 x, x)}^{\prime}\left(\frac{(1-2 \alpha) t}{2^{n} \alpha^{n}}\right) .
\end{aligned}
$$

Since $\lim _{n \rightarrow \infty} \mu_{\varphi(x, 2 x, x)}^{\prime}\left(\frac{(1-2 \alpha) t}{2^{n} \alpha^{n}}\right)=1$. Therefore, we have $\mu_{\Im(x)-\Re(x)}(t)=1$, for all $t>0$, and so $\Im(x)=\mathfrak{R}(x)$. This completes the proof. 
Corollary 3.1 Let $X$ be a real normed linear space, $\left(Z, \mu^{\prime}, \min \right)$ be an RN-space and $(Y, \mu, \min )$ be a complete $R N$-space. Let $r$ be a positive real number with $r>1, z_{0} \in Z$ and $f: X \rightarrow Y$ be a mapping satisfying

$$
\mu_{2 f\left(\frac{x+y+z}{2}\right)-f(x)-f(y)-f(z)}(t) \geq \mu_{\left(\|x\|^{r}+\|y\|^{r}+\|z\|^{r}\right) z_{0}}^{\prime}(t),
$$

for all $x, y \in X$ and $t>0$. Then the limit $\Im(x)=\lim _{n \rightarrow \infty} 2^{n} f\left(\frac{x}{2^{n}}\right)$ exists, for all $x \in X$, and defines a unique CJA mapping $\Im: X \rightarrow Y$ such that

$$
\mu_{f(x)-\Im(x)}(t) \geq \mu_{\|x\| p_{z_{0}}^{\prime}}^{\prime}\left(\frac{\left(2^{r}-2\right) t}{2^{r}+2}\right)
$$

for all $x \in X$ and $t>0$.

Proof Let $\alpha=2^{-r}$ and $\varphi: X^{3} \rightarrow Z$ be a mapping defined by $\varphi(x, y, z)=\left(\|x\|^{r}+\|y\|^{r}+\|z\|^{r}\right) z_{0}$. Then, from Theorem 3.1, the conclusion follows.

Theorem 3.2 Let $X$ be a real linear space, $\left(Z, \mu^{\prime}, \min \right)$ be an $R N$-space and $\varphi: X^{3} \rightarrow Z$ be a function such that there exists $0<\alpha<2$ such that $\mu_{\varphi(2 x, 2 y, 2 z)}^{\prime}(t) \geq \mu_{\alpha \varphi(x, y, z)}^{\prime}(t)$, for all $x \in X$ and $t>0$, and

$$
\lim _{n \rightarrow \infty} \mu_{\varphi\left(2^{n} x, 2^{n} y, 2^{n} z\right)}^{\prime}\left(2^{n} t\right)=1,
$$

for all $x, y, z \in X$ and $t>0$. Let $(Y, \mu, \min )$ be a complete $R N$-space. If $: X \rightarrow Y$ be a mapping satisfying (3.2). Then the limit $\Im(x)=\lim _{n \rightarrow \infty} \frac{f\left(2^{n} x\right)}{2^{n}}$ exists, for all $x \in X$, and defines a unique CJA mapping $\Im: X \rightarrow Y$ such that

$$
\mu_{f(x)-\Im(x)}(t) \geq \mu_{\varphi(x, 2 x, x)}^{\prime}((2-\alpha) t)
$$

for all $x \in X$ and $t>0$.

Proof It follows from (3.4) that

$$
\mu_{\frac{f(2 x)}{2}-f(x)}(t) \geq \mu_{\varphi(x, 2 x, x)}^{\prime}(2 t) .
$$

Replacing $x$ by $2^{n} x$ in (3.11), we obtain

$$
\mu_{\frac{f\left(2^{n+1} x\right)}{2^{n+1}}-\frac{f\left(2^{n} x\right)}{2^{n}}}(t) \geq \mu_{\varphi\left(2^{n} x, 2^{n+1} x, 2^{n} x\right)}^{\prime}\left(2^{n+1} t\right) \geq \mu_{\varphi(x, 2 x, x)}\left(\frac{2^{n+1} t}{\alpha^{n}}\right) .
$$

The rest of the proof is similar to the proof of Theorem 3.1.

Corollary 3.2 Let $X$ be a real normed linear space, $\left(Z, \mu^{\prime}, \min \right)$ be an $R N$-space and $(Y, \mu, \min )$ be a complete RN-space. Let $r$ be a positive real number with $0<r<1, z_{0} \in Z$ and $f: X \rightarrow Y$ be a mapping satisfying (3.9). Then the limit $\Im(x)=\lim _{n \rightarrow \infty} \frac{f\left(2^{n} x\right)}{2^{n}}$ exists, for all $x \in X$, and defines a unique CJA mapping $\mathfrak{\Im}: X \rightarrow Y$ such that

$$
\mu_{f(x)-\Im(x)}(t) \geq \mu_{\|x\|^{p} z_{0}}^{\prime}\left(\frac{\left(2-2^{r}\right) t}{2^{r}+2}\right)
$$

for all $x \in X$ and $t>0$. 
Proof Let $\alpha=2^{r}$ and $\varphi: X^{3} \rightarrow Z$ be a mapping defined by $\varphi(x, y, z)=\left(\|x\|^{r}+\|y\|^{r}+\|z\|^{r}\right) z_{0}$. Then, from Theorem 3.2, the conclusion follows.

Theorem 3.3 Let $X$ be a linear space, $\left(Y, \mu, T_{M}\right)$ be a complete $R N$-space and $\Phi$ be a mapping from $X^{3}$ to $D^{+}\left(\Phi(x, y, z)\right.$ is denoted by $\left.\Phi_{x, y, z}\right)$ such that there exists $0<\alpha<\frac{1}{2}$ such that

$$
\Phi_{2 x, 2 y, 2 z}(t) \leq \Phi_{x, y, z}(\alpha t)
$$

for all $x, y, z \in X$ and $t>0$. Let $f: X \rightarrow Y$ be a mapping satisfying

$$
\mu_{2 f\left(\frac{x+y+z}{2}\right) f(x)-f(y)-f(z)}(t) \geq \Phi_{x, y, z}(t)
$$

for all $x, y, z \in X$ and $t>0$. Then, for all $x \in X, \mathfrak{\Im}(x):=\lim _{n \rightarrow \infty} 2^{n} f\left(\frac{x}{2^{n}}\right)$ exists and $\mathfrak{\Im}: X \rightarrow Y$ is a unique CJA mapping such that

$$
\mu_{f(x)-\Im(x)}(t) \geq \Phi_{x, 2 x, x}\left(\frac{(1-2 \alpha) t}{\alpha}\right),
$$

for all $x \in X$ and $t>0$.

Proof Putting $y=2 x$ and $z=x$ in (3.13), we have

$$
\mu_{2 f\left(\frac{x}{2}\right)-f(x)}(t) \geq \Phi_{x, 2 x, x}\left(\frac{t}{\alpha}\right),
$$

for all $x \in X$ and $t>0$. Consider the set $S:=\{g: X \rightarrow Y\}$ and the generalized metric $d$ in $S$ defined by

$$
d(f, g)=\inf _{u \in(0, \infty)}\left\{\mu_{g(x)-h(x)}(u t) \geq \Phi_{x, 2 x, x}(t), \forall x \in X, t>0\right\}
$$

where inf $\emptyset=+\infty$. It is easy to show that $(S, d)$ is complete (see [39], Lemma 2.1). Now, we consider a linear mapping $J:(S, d) \rightarrow(S, d)$ such that

$$
\operatorname{Jh}(x):=2 h\left(\frac{x}{2}\right)
$$

for all $x \in X$. First, we prove that $J$ is a strictly contractive mapping with the Lipschitz constant $2 \alpha$. In fact, let $g, h \in S$ be such that $d(g, h)<\varepsilon$. Then we have $\mu_{g(x)-h(x)}(\varepsilon t) \geq \Phi_{x, 2 x, x}(t)$, for all $x \in X$ and $t>0$, and so

$$
\begin{aligned}
\mu_{J g(x)-J h(x)}(2 \alpha \varepsilon t) & =\mu_{2 g\left(\frac{x}{2}\right)-2 h\left(\frac{x}{2}\right)}(2 \alpha \varepsilon t)=\mu_{g\left(\frac{x}{2}\right)-h\left(\frac{x}{2}\right)}(\alpha \varepsilon t) \\
& \geq \Phi_{\frac{x}{2}, x, \frac{x}{2}}(\alpha t) \\
& \geq \Phi_{x, 2 x, x}(t)
\end{aligned}
$$

for all $x \in X$ and $t>0$. Thus $d(g, h)<\varepsilon$ implies that $d(J g, J h)<2 \alpha \varepsilon$. This means that $d(J g, J h) \leq 2 \alpha d(g, h)$, for all $g, h \in S$. It follows from (3.15) that $d(f, J f) \leq \alpha$. By Theorem 1.3, there exists a mapping $A: X \rightarrow Y$ satisfying the following: 
(1) $\Im$ is a fixed point of $J$, that is,

$$
\Im\left(\frac{x}{2}\right)=\frac{1}{2} \Im(x),
$$

for all $x \in X$. The mapping $\Im$ is a unique fixed point of $J$ in the set $\Omega=\{h \in S: d(g, h)<\infty\}$. This implies that $\Im$ is a unique mapping satisfying (3.18) such that there exists $u \in(0, \infty)$ satisfying $\mu_{f(x)-\Im(x)}(u t) \geq \Phi_{x, 2 x, x}(t)$, for all $x \in X$ and $t>0$.

(2) $d\left(J^{n} f, \Im\right) \rightarrow 0$ as $n \rightarrow \infty$. This implies the equality $\lim _{n \rightarrow \infty} 2^{n} f\left(\frac{x}{2^{n}}\right)=\Im(x)$, for all $x \in X$.

(3) $d(f, \Im) \leq \frac{d(f, f f)}{1-2 \alpha}$ with $f \in \Omega$, which implies the inequality $d(f, \Im) \leq \frac{\alpha}{1-2 \alpha}$ and so

$$
\mu_{f(x)-\Im(x)}(t) \geq \Phi_{x, 2 x, x}\left(\frac{(1-2 \alpha) t}{\alpha}\right)
$$

for all $x \in X$ and $t>0$. This implies that the inequality (3.14) holds. On the other hand

$$
\mu_{2^{n+1} f\left(\frac{x+y+z}{2^{n+1}}\right)-2^{n} f\left(\frac{x}{2^{n}}\right)-2^{n} f\left(\frac{y}{2^{n}}\right)-2^{n} f\left(\frac{z}{2^{n}}\right)}(t) \geq \Phi_{\frac{x}{2^{n}}, \frac{y}{2^{n}}, \frac{z}{2^{n}}}\left(\frac{t}{2^{n}}\right),
$$

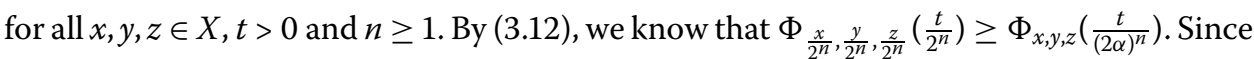
$\lim _{n \rightarrow \infty} \Phi_{x, y, z}\left(\frac{t}{(2 \alpha)^{n}}\right)=1$, for all $x, y, z \in X$ and $t>0$, we have $\mu_{2 \Im\left(\frac{x+y+z}{2}\right)-\Im(x)-\Im(y)-\Im(z)}(t)=1$, for all $x, y, z \in X$ and $t>0$. Thus the mapping $\Im: X \rightarrow Y$ satisfying (1.1). Furthermore

$$
\begin{aligned}
\Im(2 x)-2 \Im(x) & =\lim _{n \rightarrow \infty} 2^{n} f\left(\frac{x}{2^{n-1}}\right)-2 \lim _{n \rightarrow \infty} 2^{n} f\left(\frac{x}{2^{n}}\right) \\
& =2\left[\lim _{n \rightarrow \infty} 2^{n-1} f\left(\frac{x}{2^{n-1}}\right)-\lim _{n \rightarrow \infty} 2^{n} f\left(\frac{x}{2^{n}}\right)\right] \\
& =0 .
\end{aligned}
$$

This completes the proof.

Corollary 3.3 Let $X$ be a real normed space, $\theta \geq 0$ and $r$ be a real number with $r>1$. Let $f: X \rightarrow Y$ be a mapping satisfying

$$
\mu_{2 f\left(\frac{x+y+z}{2}\right)-f(x)-f(y)-f(z)}(t) \geq \frac{t}{t+\theta\left(\|x\|^{r}+\|y\|^{r}+\|z\|^{r}\right)},
$$

for all $x, y, z \in X$ and $t>0$. Then $\Im(x)=\lim _{n \rightarrow \infty} 2^{n} f\left(\frac{x}{2^{n}}\right)$ exists, for all $x \in X$, and $\Im: X \rightarrow Y$ is a unique CJA mapping such that

$$
\mu_{f(x)-\Im(x)}(t) \geq \frac{\left(2^{r}-2\right) t}{\left(2^{r}-2\right) t+\left(2^{r}+2\right) \theta\|x\|^{r}},
$$

for all $x \in X$ and $t>0$.

Proof The proof follows from Theorem 3.3 if we take $\Phi_{x, y, z}(t)=\frac{t}{t+\theta\left(\|x\|^{r}+\|y\|^{r}+\|z\|^{r}\right)}$, for all $x, y, z \in X$ and $t>0$. In fact, if we choose $\alpha=2^{-r}$, then we get the desired result. 
Theorem 3.4 Let $X$ be a linear space, $\left(Y, \mu, T_{M}\right)$ be a complete $R N$-space and $\Phi$ be a mapping from $X^{3}$ to $D^{+}\left(\Phi(x, y, z)\right.$ is denoted by $\left.\Phi_{x, y, z}\right)$ such that for some $0<\alpha<2$, $\Phi_{\frac{x}{2}, \frac{y}{2}, \frac{z}{2}}(t) \leq \Phi_{x, y, z}(\alpha t)$, for all $x, y, z \in X$ and $t>0$. Let $f: X \rightarrow Y$ be a mapping satisfying (3.13). Then the limit $\Im(x):=\lim _{n \rightarrow \infty} \frac{f\left(2^{n} x\right)}{2^{n}}$ exists, for all $x \in X$, and $\Im: X \rightarrow Y$ is a unique CJA mapping such that

$$
\mu_{f(x)-\Im(x)}(t) \geq \Phi_{x, 2 x, x}((2-\alpha) t)
$$

for all $x \in X$ and $t>0$.

Proof Putting $y=2 x$ and $z=x$ in (3.13), we have

$$
\mu_{\frac{f(2 x)}{2}-f(x)}(t) \geq \Phi_{x, 2 x, x}(2 t)
$$

for all $x \in X$ and $t>0$. Let $(S, d)$ be the generalized metric space defined in the proof of Theorem 3.1. Now, we consider a linear mapping $J:(S, d) \rightarrow(S, d)$ such that $J h(x):=$ $\frac{1}{2} h(2 x)$, for all $x \in X$. It follows from (3.21) that $d(f, J f) \leq \frac{1}{2}$. By Theorem 1.3 , there exists a mapping $\mathfrak{s}: X \rightarrow Y$ satisfying the following:

(1) $\mathfrak{s}$ is a fixed point of $J$, that is,

$$
\Im(2 x)=2 \Im(x),
$$

for all $x \in X$. The mapping $\Im$ is a unique fixed point of $J$ in the set $\Omega=\{h \in S: d(g, h)<\infty\}$. This implies that $\mathfrak{s}$ is a unique mapping satisfying (3.22) such that there exists $u \in(0, \infty)$ satisfying $\mu_{f(x)-\Im(x)}(u t) \geq \Phi_{x, 2 x, x}(t)$, for all $x \in X$ and $t>0$.

(2) $d\left(J^{n} f, \mathfrak{I}\right) \rightarrow 0$ as $n \rightarrow \infty$. This implies the equality

$$
\lim _{n \rightarrow \infty} \frac{f\left(2^{n} x\right)}{2^{n}}=\Im(x)
$$

for all $x \in X$.

(3) $d(f, \Im) \leq \frac{d(f, J f)}{1-\frac{\alpha}{2}}$ with $f \in \Omega$, which implies the inequality $\mu_{f(x)-\Im(x)}\left(\frac{t}{2-\alpha}\right) \geq \Phi_{x, 2 x, x}(t)$, for all $x \in X$ and $t>0$. This implies that the inequality (3.20) holds. The rest of the proof is similar to the proof of Theorem 3.3.

Corollary 3.4 Let $X$ be a real normed space, $\theta \geq 0$ and $r$ be a real number with $0<r<1$. Let $f: X \rightarrow Y$ be a mapping satisfying (3.19). Then the limit $\Im(x)=\lim _{n \rightarrow \infty} \frac{f\left(2^{n} x\right)}{2^{n}}$ exists, for all $x \in X$, and $\mathfrak{s}: X \rightarrow Y$ is a unique CJA mapping such that

$$
\mu_{f(x)-\Im(x)}(t) \geq \frac{\left(2-2^{r}\right) t}{\left(2-2^{r}\right) t+\left(2^{r}+2\right) \theta\|x\|^{r}},
$$

for all $x \in X$ and $t>0$.

Proof The proof follows from Theorem 3.4 if we take $\Phi_{x, y, z}(t)=\frac{t}{t+\theta\left(\|x\|^{r}+\|y\|^{r}+\|z\|^{r}\right)}$, for all $x, y, z \in X$ and $t>0$. In fact, if we choose $\alpha=2^{r}$, then we get the desired result. 


\section{Competing interests}

The authors declare that they have no competing interests.

\section{Authors' contributions}

All authors conceived of the study, participated in its design and coordination, drafted the manuscript, participated in the sequence alignment, and read and approved the final manuscript.

\section{Author details}

'Department of Mathematics, Research Institute for Natural Sciences, Hanyang University, Seoul, 133-791, Korea.

${ }^{2}$ Department of Mathematics, Beyza Branch, Islamic Azad University, Beyza, Iran.

\section{Received: 30 August 2012 Accepted: 26 March 2014 Published: 23 May 2014}

\section{References}

1. Ulam, SM: Problems in Modern Mathematics. Science Editions, Wiley, New York (1964)

2. Hyers, DH: On the stability of the linear functional equation. Proc. Natl. Acad. Sci. USA 27, 222-224 (1941)

3. Aoki, T: On the stability of the linear transformation in Banach spaces. J. Math. Soc. Jpn. 2, $64-66$ (1950)

4. Rassias, TM: On the stability of the linear mapping in Banach spaces. Proc. Am. Math. Soc. 72, 297-300 (1978)

5. Găvruta, P: A generalization of the Hyers-Ulam-Rassias stability of approximately additive mappings. J. Math. Anal. Appl. 184, 431-436 (1994)

6. Skof, F: Local properties and approximation of operators. Rend. Semin. Mat. Fis. Milano 53, 113-129 (1983)

7. Cholewa, PW: Remarks on the stability of functional equations. Aequ. Math. 27, 76-86 (1984)

8. Czerwik, S: Functional Equations and Inequalities in Several Variables. World Scientific, River Edge (2002)

9. Arriola, LM, Beyer, WA: Stability of the Cauchy functional equation over $p$-adic fields. Real Anal. Exch. 31, 125-132 (2005/06)

10. Azadi Kenary, H, Lee, J, Park, C: Non-Archimedean stability of an AQQ functional equation. J. Comput. Anal. Appl. 14, 211-227 (2012)

11. Azadi Kenary, H, Park, C, Lee, S: On the fuzzy stability of a generalized Jensen quadratic functional equation. J. Comput. Anal. Appl. 14, 1118-1129 (2012)

12. Azadi Kenary, H, Jang, S, Park, C: Approximation of an additive-quadratic functional equation in RN-spaces. J. Comput. Anal. Appl. 14, 1190-1209 (2012)

13. Eshaghi Gordji, M, Abbaszadeh, S, Park, C: On the stability of a generalized quadratic and quartic type functional equation in quasi-Banach spaces. J. Inequal. Appl. 2009, Article ID 153084 (2009)

14. Eshaghi Gordji, M, Bavand Savadkouhi, M: Stability of mixed type cubic and quartic functional equations in random normed spaces. J. Inequal. Appl. 2009, Article ID 527462 (2009)

15. Eshaghi Gordji, M, Bavand Savadkouhi, M, Park, C: Quadratic-quartic functional equations in RN-spaces. J. Inequal. Appl. 2009, Article ID 868423 (2009)

16. Eshaghi Gordji, M, Khodaei, H, Khodabakhsh, R: General quartic-cubic-quadratic functional equation in non-Archimedean normed spaces. Sci. Bull. "Politeh." Univ. Buchar., Ser. A, Appl. Math. Phys. 72(3), 69-84 (2010)

17. Eshaghi Gordji, M, Savadkouhi, MB: Stability of cubic and quartic functional equations in non-Archimedean spaces. Acta Appl. Math. 110, 1321-1329 (2010)

18. Eshaghi Gordji, M, Savadkouhi, MB: Stability of a mixed type cubic-quartic functional equation in non-Archimedean spaces. Appl. Math. Lett. 23, 1198-1202 (2010)

19. Khodaei, H, Rassias, TM: Approximately generalized additive functions in several variables. Int. J. Nonlinear Anal. Appl. $1,22-41(2010)$

20. Mohammadi, M, Cho, Y, Park, C, Vetro, P, Saadati, R: Random stability of an additive-quadratic-quartic functional equation. J. Inequal. Appl. 2010, Article ID 754210 (2010)

21. Park, C: Fixed points and Hyers-Ulam-Rassias stability of Cauchy-Jensen functional equations in Banach algebras. Fixed Point Theory Appl. 2007, Article ID 50175 (2007)

22. Park, C: Generalized Hyers-Ulam-Rassias stability of quadratic functional equations: a fixed point approach. Fixed Point Theory Appl. 2008, Article ID 493751 (2008)

23. Rassias, TM: Functional Equations, Inequalities and Applications. Kluwer Academic, Dordrecht (2003)

24. Rassias, TM: On the stability of the quadratic functional equation and its applications. Stud. Univ. Babeş-Bolyai, Math. XLIII, 89-124 (1998)

25. Rassias, TM: The problem of S.M. Ulam for approximately multiplicative mappings. J. Math. Anal. Appl. 246, 352-378 (2000)

26. Rassias, TM: On the stability of functional equations in Banach spaces. J. Math. Anal. Appl. 251, 264-284 (2000)

27. Saadati, R, Park, C: Non-Archimedean $\mathcal{L}$-fuzzy normed spaces and stability of functional equations. Comput. Math. Appl. 60, 2488-2496 (2010)

28. Saadati, R, Vaezpour, M, Cho, Y: A note to paper 'On the stability of cubic mappings and quartic mappings in random normed spaces'. J. Inequal. Appl. 2009, Article ID 214530 (2009)

29. Saadati, R, Zohdi, MM, Vaezpour, SM: Nonlinear L-random stability of an ACQ-functional equation. J. Inequal. Appl. 2011, Article ID 194394 (2011)

30. Hensel, K: Uber eine neue Begrundung der Theorie der algebraischen Zahlen. Jahresber. Dtsch. Math.-Ver. 6, 83-88 (1897)

31. Deses, D: On the representation of non-Archimedean objects. Topol. Appl. 153, 774-785 (2005)

32. Katsaras, AK, Beoyiannis, A: Tensor products of non-Archimedean weighted spaces of continuous functions. Georgian Math. J. 6, 33-44 (1999)

33. Khrennikov, A: Non-Archimedean Analysis: Quantum Paradoxes, Dynamical Systems and Biological Models Mathematics and Its Applications, vol. 427. Kluwer Academic, Dordrecht (1997)

34. Kominek, Z: On a local stability of the Jensen functional equation. Demonstr. Math. 22, 499-507 (1989)

35. Nyikos, PJ: On some non-Archimedean spaces of Alexandrof and Urysohn. Topol. Appl. 91, 1-23 (1999) 
36. Schewizer, B, Sklar, A: Probabilistic Metric Spaces. North-Holland Series in Probability and Applied Mathematics. North-Holland, New York (1983)

37. Cădariu, L, Radu, V: Fixed points and the stability of Jensen's functional equation. J. Inequal. Pure Appl. Math. 4(1), Article ID 4 (2003)

38. Radu, $\mathrm{V}$ : The fixed point alternative and the stability of functional equations. Fixed Point Theory 4, $91-96$ (2003)

39. Mihet, $D$, Radu, $V$ : On the stability of the additive Cauchy functional equation in random normed spaces. J. Math. Anal. Appl. 343, 567-572 (2008)

10.1186/1029-242X-2014-209

Cite this article as: Park et al.: Non-Archimedean and random HUR-approximation of a Cauchy-Jensen additive mapping. Journal of Inequalities and Applications 2014, 2014:209

\section{Submit your manuscript to a SpringerOpen ${ }^{\odot}$} journal and benefit from:

- Convenient online submission

Rigorous peer review

- Immediate publication on acceptance

- Open access: articles freely available online

- High visibility within the field

- Retaining the copyright to your article 